\title{
Leishmaniasis-Associated Hemophagocytic Syndrome Revisited: Not an Uncommon Clinical Presentation of Leishmaniasis
}

\author{
Asterios Saitis $^{\mathrm{a}}$, Nikolaos K. Gatselis ${ }^{\mathrm{a}, \mathrm{b}}$, George N. Dalekos ${ }^{\mathrm{a}}$
}

\begin{abstract}
Visceral leishmaniasis (VL) is an uncommon but important cause of reactive hemophagocytic syndrome (HS) that should be seriously considered in patients coming from endemic areas. We describe two cases of VL-associated HS; a young woman with fever, cytopenias and hyperferritinemia and an immunocompromised patient with fever, splenomegaly, hyperferritinemia and pancytopenia. The diagnosis of leishmaniasis was established with polymerase chain reaction for leishmania and bone marrow examination, whereas leihmaniasis specific treatment with liposomal amphotericin led to full clinical recovery and complete remission of HS. From the clinical point of view, it should be emphasized that the high clinical suspicion along with the use of modern, high yield diagnostic tools, may lead to early diagnosis of VL-associated HS, minimizing unnecessary hospitalization and potentially harmful investigations and treatments.
\end{abstract}

Keywords: Visceral leishmaniasis; Hemophagocytosis; Hemophagocytic syndrome; Immunosuppression

\section{Introduction}

Hemophagocytic syndrome (HS) is an uncommon but lifethreatening condition characterized by high fever, lymphadenopathy, hepatosplenomegaly, cytopenias, liver dysfunction and hyperferritinemia. It is caused by a dysregulation in natural killer cell function, leading to an uncontrolled activation of histiocytes/macrophages and lymphocytes, cytokine

\footnotetext{
Manuscript accepted for publication May 24, 2012

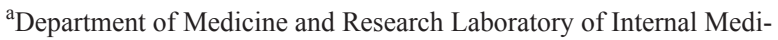
cine, Thessaly University, Medical School, Larissa, Thessaly, Greece ${ }^{\mathrm{b}}$ Corresponding author: Nikolaos K. Gatselis, Lecturer in Medicine, Department of Medicine and Research Laboratory of Internal Medicine, University of Thessaly, School of Medicine, Biopolis, Larissa, 41110 Larissa, Greece. Email: ngatsel@med.uth.gr
}

doi: $10.4021 / \mathrm{jmc} 715 \mathrm{~W}$ overproduction and hemophagocytosis [1]. HS can be either primary, due to genetic mutations, or secondary, associated with malignancies, autoimmune diseases and infections. Among the infectious agents, Epstein Barr Virus (EBV) is the most common cause of the syndrome. However, HS can also be associated with other viral infections (other herpes viruses, HIV, influenza, parvovirus, hepatitis viruses) as well as with bacterial, fungal and parasitic infections, including visceral leishmaniasis (VL) in endemic areas [2].

Herein, we describe two cases of VL-associated HS, followed by a short review of the literature.

\section{Case Report}

\section{Patient 1}

A 22-years-old previously healthy woman was admitted to our department with a 4-day history of fever up to $39{ }^{\circ} \mathrm{C}$, malaise, anorexia and nausea. She also mentioned several episodes of frontal headache without neck stiffness or photophobia, arthralgias, as well as an episode of epistaxis.

The physical examination revealed only mild splenomegaly, while the routine laboratory tests revealed leukopenia $(3,500 / \mu \mathrm{L}$; normal range $4,000-10,000 / \mu \mathrm{L})$, thrombocytopenia $(75,000 / \mu \mathrm{L}$; normal range $150,000-400,000 / \mu \mathrm{L})$, an increase of lactate dehydrogenase (LDH $556 \mathrm{IU} / \mathrm{L}$; upper normal limit (UNL) $230 \mathrm{IU} / \mathrm{L}$ ) and creatinine phosphokinase (CPK 273 IU/L; UNL 145 IU/L). A chest radiograph was normal, while electrocardiography revealed sinus tachycardia.

During the first week of hospitalization an extensive diagnostic work-up was performed. Blood, urine, bone marrow and cerebrospinal fluid cultures were sterile, whereas serological studies for Brucella, Leishmania, Leptospira, Enteroviruses, Adenoviruses, hepatitis A, B, C viruses and HIV infection tested negative. Testing for EBV and other herpes viruses were consistent with past infection and serum polymerase chain reaction (PCR) for EBV and CMV DNA was negative. Immunological tests (antinuclear antibodies, anti-neutrophil cytoplasmic antibodies, rheumatoid factors) were also negative. 
The patient initially received ceftriaxone empirically. However, fever and the other symptoms persisted whereas laboratory tests showed deterioration of the hematological parameters. On clinical suspicion of HS, biochemical and coagulation tests were performed that revealed an extremely high level of ferritin $(24,000 \mathrm{ng} / \mathrm{mL}$; normal range 20 - 400 $\mathrm{ng} / \mathrm{mL}$ ) and a mild decrease of fibrinogen. Myelogram confirmed extensive hemophagocytosis. A diagnosis of HS was established, as 6 out of 8 diagnostic criteria for HS were fulfilled; fever, splenomegaly, cytopenias, hyperferritinemia, hypofibrinogenemia and hemophagocytosis in the bone marrow [3].

HS was initially attributed to adult Still's disease, as the majority of the infectious, immunological and neoplastic diseases related to HS had been ruled out by the aforementioned tests. So, the initial treatment consisted of corticosteroids and gamma globulin infusions as we have described recently [4]. However, from the clinical and laboratory points of view, the patient deteriorated further.

Treatment failure led to a reconsideration of the diagnosis. Other causes of HS were considered, including VL, an endemic parasitosis in Greece that can be manifested as HS. Peripheral blood PCR for Leishmania donovani was positive, while a second bone marrow aspiration revealed Leismania parasites in macrophage cytoplasm. As the diagnosis of VL-associated HS had been established, the patient received liposomal amphotericin (daily dose $4 \mathrm{mg} / \mathrm{kg}$ body weight on days 1 - 5, 14 and 21) leading to a full clinical recovery and restoration of hematological parameters. The patient has been on follow-up by our department for more than 2 years, and no relapse has been noticed.

\section{Patient 2}

A 40-years-old man was admitted to our department due to 20 -days history of high fever and cytopenias. The patient suffered from myasthenia gravis, having a close follow-up by the neurology department. He was under immunosuppressive treatment with prednisone and azathioprine combined with gamma-globulin infusions. The patient had been in his usual state of health until 20 days before admission, when he started having everyday fever up to $40{ }^{\circ} \mathrm{C}$ along with night sweats, anorexia and weight loss.

Physical examination revealed a $2 / 6$ systolic murmur at the right upper sternal border and splenomegaly. On initial laboratory tests, the total blood count revealed pancytopenia; white cell count $2,300 / \mu \mathrm{L}$, hemoglobin $8 \mathrm{gr} / \mathrm{dL}$ (normal range 12 - $16 \mathrm{gr} / \mathrm{dL}$ ), platelet count $75,000 / \mu \mathrm{L}$. Biochemical tests showed mild transaminasemia (aspartate transaminase and alanine transaminase levels of 52 and $43 \mathrm{IU} / \mathrm{L}$ respectively; normal range $10-35 \mathrm{U} / \mathrm{L})$, a moderate increase of inflammation markers (C-reactive protein $11.3 \mathrm{mg} / \mathrm{dL}$; UNL $0.5 \mathrm{mg} / \mathrm{dL})$ and significant hyperferritinemia $(6,329 \mathrm{ng} / \mathrm{mL})$ and hypergammaglobulinemia (4.5 g/dL; UNL $3.5 \mathrm{~g} / \mathrm{dL})$.
The differential diagnosis included the common infectious and neoplastic causes of febrile splenomegaly. Subsequently, an extensive investigation was performed that ruled out infective endocarditis, brucellosis, leptospirosis, EBV, CMV, HIV or Parvo-virus infection as well as lymphoproliferative disorders. However, due to the typical findings such as fever, splenomegaly, pancytopenia, hypergammaglobulinemia and history of immunosuppression, VL was considered as probable diagnosis. Therefore, peripheral blood and bone marrow PCR for leishmania DNA was performed that were positive, whereas myelogram revealed the presence of leishmania parasites in macrophage cytoplasm, establishing the diagnosis of leishmaniasis. Furthermore, according to the aferomentioned criteria of HS [3], a diagnosis of VLassociated HS was established since 5 out of 8 criteria for HS diagnosis were fulfilled [3]; fever, splenomegaly, cytopenias, hyperferritinemia, low natural killer activity as estimated by immunophenotyping.

As the patient was immunocompromised, he received an intensive regimen of liposomal amphotericin (daily dose of $4 \mathrm{mg} / \mathrm{kg}$ body weight on days $1-5,10,17,24,31,38)$ to prevent a possible relapse. Specific treatment resulted in full resolution of patient's symptoms and signs as well as complete remission of HS.

However, despite the intensive treatment, patient's symptoms reemerged a few months later and a relapse of VL was confirmed by peripheral blood and bone marrow PCR for leishmania. A second intensive regimen of liposomal amphotericin was administered, followed by prophylactic monthly doses of liposomal amphotericin ( $4 \mathrm{mg} / \mathrm{kg}$ body weight) to prevent new relapses. Since then, the patient is free of symptoms for 8 months while hematological abnormalities have been fully restored.

\section{Discussion}

We described two cases of VL-associated HS from Greece, a country endemic for this parasitosis. To the best of our knowledge, this is the second report of secondary HS due to VL reported from Greece, the first one having been published more than ten years ago [5].

The association of VL with HS has been known since the late 70s, when a first case of VL mimicking histiocytosis was reported [6]. Since then, only a few cases of HS secondary to VL have been reported, and the majority of them were infants or children living in endemic areas [7]. However, HS as a pathologic finding in VL might be quite more common. In a recent case series examining the hematological features of the bone marrow examination associated with VL, HS was found in 12 out of $16(75 \%)$ patients examined [8].

The recognition of HS can be a diagnostic challenge, as clearly demonstrated in the first case described. According to a recent systematic review of VL-associated HS, the 
mean delay in the diagnosis was 9 weeks [7]. This delay is attributed to the overlapping clinical features between VL and primary histiocytosis, as well as to the low sensitivity of diagnostic methods for VL, such as bone marrow examination and serologic testing. The moderate yield of the "traditional' diagnostic tools may hinder the diagnosis. In the same systematic review of Rajagopala et al, the first bone marrow aspiration failed to establish the presence of Leismania donovani bodies at onset in $36.3 \%$ of cases examined, while the diagnostic yield of serology was also low (23.5\%) [7]. In another French pediatric series of VL, the parasite was not detected in $22 \%$ of the patients, whereas there were cases with Leismania donovani bodies recognized on the fifth bone marrow aspiration $[9,10]$. These diagnostic difficulties are confirmed in the first patient of the present article, where both bone marrow aspiration and serology were initially negative, leading to further investigations and potentially harmful treatment. Therefore, on a strong clinical suspicion for VL-associated HS, a repeated bone marrow evaluation, and especially the use of highly specific and sensitive PCR assays for leishmania might be necessary [11].

The second patient highlights the increasing problem of opportunistic intracellular infections in immunocompromised patients, as a result of the HIV epidemic and the widespread use of enhanced immunosuppressant therapy for various diseases. It has been estimated that HIV related immunosuppression increases the risk of reactivating $\mathrm{VL}$ by $100-1,000$ times [12], while there are many cases of disseminated VL in immunocompromised patients due to corticosteroid therapy, immunosuppression after solid organ transplantation, or hematologic neoplasia [13, 14]. However, HS is probably an atypical manifestation of VL in immunocompromised patients, as till now only two cases of VLassociated HS in immunocompromised patients (one due to immunosuppressant therapy for Harada's disease and the other due to HIV infection) have been reported $[15,16]$.

Our cases illustrate the therapeutic challenges of VLassociated HS, both in immunocompetent and immunocompromised patients. Although immunosuppression schedules including etoposide regimen [3] or combination treatment with corticosteroids and intravenous immunoglobulin infusions [4] are used for life-threatening infectious-related HS, the case of VL-associated HS is probably the only type of reactive HS where pathogen-specific treatment is sufficient to control the disease [2]. On the other hand, if the diagnosis of VL eludes, the use of immunosuppressant and chemotherapeutic drugs usually administered for familial and other reactive types of HS, may lead to a dramatic clinical and hematological deterioration, as illustrated in our first case. Therefore, VL should be ruled out in all patients with HS living in endemic areas before immunosuppression is started [17].

Treatment of VL in immunocompromised individuals, including the few cases manifested as HS, consist an un- resolved question, due to the poor results of conventional therapy in theses cases. Clinical failure and mortality is high, while many patients experience one or more relapses, as in our second case described [14]. Therefore, an intensive regimen of anti-parasitic treatment and especially of liposomal amphotericin [14] should be considered in any immunocompromised patient suffering from VL with or without HS. In the case of relapse despite intensive treatment, a prophylactic regimen (using amphotericin or pentamidine) is an option $[18,19]$, although there is lack of evidence from large clinical trials to support it.

\section{Conclusions}

Although traditionally VL is considered as an uncommon cause of reactive HS, this association might be quite more common [8] and due to the high mortality of HS if untreated, it should be seriously considered in patients coming from endemic areas for VL. The correct and early diagnosis will lead to pathogen-specific and highly effective treatment. Therefore, the high clinical suspicion as well as the use of modern, high yield diagnostic tools, such as PCR, may lead to early diagnosis of VL-associated HS, minimizing unnecessary hospitalization and potentially harmful investigations and treatments.

\section{Conflict of Interest}

There is no conflict of interest in this paper.

\section{Grand Support}

There is no grant for this paper.

\section{References}

1. Grom AA, Villanueva J, Lee S, Goldmuntz EA, Passo MH, Filipovich A. Natural killer cell dysfunction in patients with systemic-onset juvenile rheumatoid arthritis and macrophage activation syndrome. J Pediatr. 2003;142(3):292-296.

2. Rouphael NG, Talati NJ, Vaughan C, Cunningham K, Moreira R, Gould C. Infections associated with haemophagocytic syndrome. Lancet Infect Dis. 2007;7(12):814-822.

3. Henter JI, Horne A, Arico M, Egeler RM, Filipovich AH, Imashuku S, Ladisch S, et al. HLH-2004: Diagnostic and therapeutic guidelines for hemophagocytic lymphohistiocytosis. Pediatr Blood Cancer. 2007;48(2):124131.

4. Argyraki CK, Gabeta S, Zachou K, Boulbou M, Polyzos 
A, Dalekos GN. Favourable outcome of life-threatening infectious-related haemophagocytic syndrome after combination treatment with corticosteroids and intravenous immunoglobulin infusions. Eur J Intern Med. 2011;22(6):e155-157.

5. Kontopoulou T, Tsaousis G, Vaidakis E, Fanourgiakis P, Michalakeas E, Trigoni E, Samarkos M. Hemophagocytic syndrome in association with visceral leishmaniasis. Am J Med. 2002;113(5):439-440.

6. Matzner Y, Behar A, Beeri E, Gunders AE, Hershko C. Systemic leishmaniasis mimicking malignant histiocytosis. Cancer. 1979;43(1):398-402.

7. Rajagopala S, Dutta U, Chandra KS, Bhatia P, Varma N, Kochhar R. Visceral leishmaniasis associated hemophagocytic lymphohistiocytosis--case report and systematic review. J Infect. 2008;56(5):381-388.

8. Bhatia P, Haldar D, Varma N, Marwaha R, Varma S. A case series highlighting the relative frequencies of the common, uncommon and atypical/unusual hematological findings on bone marrow examination in cases of visceral leishmaniasis. Mediterr J Hematol Infect Dis. 2011;3(1):e2011035.

9. Gagnaire MH, Galambrun C, Stephan JL. Hemophagocytic syndrome: A misleading complication of visceral leishmaniasis in children--a series of 12 cases. Pediatrics. 2000;106(4):E58.

10. Sukova M, Stary J, Houskova J, Nohynkova E. [Hemophagocytic lymphohistiocytosis as a manifestation of visceral leishmaniasis]. Cas Lek Cesk. 2002;141(18):581584.

11. Antinori S, Calattini S, Longhi E, Bestetti G, Piolini R, Magni C, Orlando G, et al. Clinical use of polymerase chain reaction performed on peripheral blood and bone marrow samples for the diagnosis and monitoring of visceral leishmaniasis in HIV-infected and HIVuninfected patients: a single-center, 8-year experience in Italy and review of the literature. Clin Infect Dis. 2007;44(12):1602-1610.
12. Lopez-Velez R, Perez-Molina JA, Guerrero A, Baquero F, Villarrubia J, Escribano L, Bellas C, et al. Clinicoepidemiologic characteristics, prognostic factors, and survival analysis of patients coinfected with human immunodeficiency virus and Leishmania in an area of Madrid, Spain. Am J Trop Med Hyg. 1998;58(4):436-443.

13. Altozano JG, Lopez-Gomez JM, Robles R, Muino A, Romero J, Valderrabano F. [Visceral leishmaniasis in systemic lupus erythematosus]. Med Clin (Barc). 1987;88(10):417-418.

14. Fernandez-Guerrero ML, Robles P, Rivas P, Mojer F, Muniz G, de Gorgolas M. Visceral leishmaniasis in immunocompromised patients with and without AIDS: a comparison of clinical features and prognosis. Acta Trop. 2004;90(1):11-16.

15. Fiteni I, Perez-Lungmus G, Grasa J, Motis C. The hemophagocytic syndrome in an immunocompromised patient: A diagnostic challenge. Can J Infect Dis. 2004;15(2):101-103.

16. Patel KK, Patel AK, Sarda P, Shah BA, Ranjan R. Immune reconstitution visceral leishmaniasis presented as hemophagocytic syndrome in a patient with AIDS from a nonendemic area: a case report. J Int Assoc Physicians AIDS Care (Chic). 2009;8(4):217-220.

17. Makaritsis KP, Gatselis NK, Ioannou M, Petinaki E, Dalekos GN. Polyclonal hypergammaglobulinemia and high smooth-muscle autoantibody titers with specificity against filamentous actin: consider visceral leishmaniasis, not just autoimmune hepatitis. Int J Infect Dis. 2009;13(4):e157-160.

18. Patel TA, Lockwood DN. Pentamidine as secondary prophylaxis for visceral leishmaniasis in the immunocompromised host: report of four cases. Trop Med Int Health. 2009;14(9):1064-1070.

19. Borrelli P, Imperato A, Murdaca G, Scudeletti M. Liposomal amphotericin B as first line and secondary prophylactic treatment for visceral leishmaniasis in a patient infected with HIV. Ann Ital Med Int. 2000;15(2):169-171. 